\title{
Different Feeds and Feeding Regimens have an Impact on Zebrafish Larval Rearing and Breeding Performance
}

\author{
Matheus Farias ${ }^{1,2}$ and Ana C. Certal ${ }^{2 *}$ \\ ${ }^{1}$ ISPA - Instituto Universitario, Lisbon, Portugal \\ ${ }^{2}$ Champalimaud Centre for the Unknown, Lisbon, Portugal
}

Received: August 28, 2015, 2015; Accepted: December 20, 2015; Published: January 12, 2016

*Corresponding author: Ana Catarina Certal, Fish Platform, Champalimaud Centre for the Unknown; 1400-038 Lisbon, Portugal, Tel: +351 210480200; Fax: +351 210480299; E-mail: catarina.certal@fundacaochampalimaud.pt

\begin{abstract}
Zebrafish is one of the most used model organisms in biomedical sciences and other research fields. Despite the huge importance of having an efficient and high throughput zebrafish aquaculture, little is known on its specific feeding requirements and very few studies have focused on the impact of feeds on the overall fish performance throughout their lifecycle. Here we present a comparative study using three commercial dry feeds (Skretting®Gemma Micro, Zeigler®Larval AP100 and ZM®) from first feed to 180 days postfertilization (dpf) in three different feeding regimens after day 9 post-fertilization (dry food only, a combination of dry food with live food and live food only). The impact on survival, growth, age of sexual maturity, fecundity and embryo viability, was assessed in three different fish strains (wild-type, mutant and transgenic). Gemma Micro stood out as the best performer in all parameters evaluated. We also show that better results are obtained when dry feeds are complemented with live feeds, but it is possible to attain quite a satisfactory level of performance using only dry feeds in adult stages which may be relevant for many studies and facilities.
\end{abstract}

Keywords: Zebrafish; Feeding; Larval rearing; Growth; Breeding

\section{Introduction}

Zebrafish, Danio rerio (Hamilton, 1822), is a small tropical freshwater cyprinid. Its small size, external development, high fecundity, embryo transparency, short generation time, easy management, physiological similarity to mammals and the amount of research tools available, have turned this species into one of the main model organisms for developmental studies [13]. In the last two decades the use of this research model has dramatically widespread to various fields of biological sciences, being currently used in ecotoxicology [4-5], neurosciences [6-7], behavior [8-11], cancer [12-14], genetics [15], nutrition [16-20], aquaculture [19,21], as a model for human diseases [22-24], drug discovery [25], among others [26-28].
This boom in research has led its aquaculture to grow exponentially worldwide without sufficient accompanying studies on novel methods for husbandry and larval rearing which would optimize an intensive production of fish for research with adequate standardization and fish welfare. With new transgenic tools being rapidly developed, the zebrafish mutation project as well as its current role as a powerful tool in drug screens, it became essential to find better protocols and methods to breed and raise zebrafish faster, more reliably and with better welfare. However, little is known on the nutritional requirements of zebrafish $[29,30]$, being mostly reared with information available for Cyprinaformes [30,31]. This is becoming a major concern within the research community as it imposes a difficulty to standardize a husbandry protocol in different facilities [32, 33]. Proper nutrition is not only important for individual growth and survival [29] but also to the reproductive success [34] which directly affects offspring fitness [35].

Traditionally, zebrafish have been raised with a combination of live feeds and processed dry feeds. In the first 5-7 days postfertilization (dpf), larvae extract nutrients from the yolk sac not requiring exogenous feeding. After that period they are commonly fed with paramecium or rotifers until 9-15 dpf. Thereafter their diet is based on artemia (Artemia nauplii) complemented with processed dry feed [36, 37].

Despite the benefits of live feeding (high digestibility, encouragement of prey capture behavior, nutritional customization by enriching live feeds [38]), this practice can be hazardous as live organisms can work as vectors for pathogens [37-39]. On the other hand, unsupplemented live feeds alone cannot sustain zebrafish nutritional needs, being reported to reduce growth and even to cause developmental malformations [40].

Recently, some studies were published on the nutritional requirements of zebrafish with the aim of findingan optimized 
feeding protocol $[33,34,41-44]$. A major drawback of these studies is that they focused mostly on the juvenile and adult stages leaving the larval stage largely unstudied. The few studies contemplating first-feeding have used live feeding $[45,46]$, rendering few data on dry food as first-feed.

In this study we present a comparison analysis for three commercial feeds (ZM, Zeigler Larval AP100 and Skretting Gemma Micro) in three different zebrafish strains (wild-type, mutant, transgenic), under three different feeding regimens after day 9 post-fertilization (dry feed only, a combination of dry feed with live feed and live feed only) regarding growth, survival, sexual maturity, fecundity and embryo viability. The main goal was to understand which dry feed would better complement live feeding and up to a certain extent would reduce the need for live feeds, taking into account the growing demand of zebrafish facilities to decrease the fish life-cycle and increase breeding performance and welfare.

\section{Materials and Methods}

\section{Fish}

Three different zebrafish strains were used, representing the main types of fish used in labs - wild-type, mutant and transgenic fish - in order to check if there was different feeding requirements for the different strains. Representing the wild-type, $A B$ was chosen as the most common wild-type strain used in research. Representing the mutants, Nacre (mitfa -/-), a pigmentation mutant, was chosen due to its broad distribution among labs for being often used as a background strain for imaging in neuroscience and cancer research. HuC:GCaMP5G, a transgenic strain expressing the calcium indicator GCaMP5G in neurons [47] in a heterozygous background ( $\mathrm{AB} \times \mathrm{Nacre}$ ) was chosen as an example of a transgenic strain.

\section{Embryo generation}

Sexually mature zebrafish from each of the three study lines were set up to cross in pools at dawn. Eggs were collected, transferred to Petri dishes with embryo medium and kept in an incubator at $28^{\circ} \mathrm{C}$ until $6 \mathrm{dpf}$. Each dish contained groups of 50 embryos that were then vested to a $3.5 \mathrm{~L}$ tank filled with $500 \mathrm{~mL}$ of embryo medium and introduced in the main holding system with no water flow (Tecniplast ${ }^{\circledR}$ recirculating system with the following water parameters: $\mathrm{pH} 7.2 ; 28.0^{\circ} \mathrm{C} ; 1000 \mu \mathrm{s}$ ). During the following 2 days, $200 \mathrm{~mL}$ of system water was added to each tank per day. From 9 to 15 dpf, larvae were kept with system water drop-flow and afterwards started on constant flow.

\section{Feeding assays}

Tanks from each line were assigned to nine experimental groups according to the diet and regimen Table 1. For each group there were two replicate tanks.

All tanks were fed four times per day in working days and once a day in weekends and holidays, always at the same hour of the day. In working days, tanks from the dry live groups were fed 2 times Artemia +2 times dry feed. In the weekends and holidays, tanks from dry only and dry live groups were fed only dry food whereas tanks from the control group were fed only Artemia. The first two feed sizes (up to $30 \mathrm{dpf}$ - Skretting ${ }^{\circledR}$ Gemma Micro 75 and 150; Zeigler ${ }^{\circledR}$ Larval AP100<100 and 100-150; ZM ${ }^{\circledR} 00$ and 100 ) were dispensed with a $1000 \mu \mathrm{L}$ pipette tip, one dip tip per feeding. Subsequent feed sizes (After $30 \mathrm{dpf}$ - Skretting ${ }^{\circledR}$ Gemma Micro 150; Zeigler ${ }^{\circledR}$ Larval AP100 150-250; ZM ${ }^{\circledR} 200$ ) were given with a disposable Pasteur pipette tip, one dip tip per feeding. Freshly hatched decapsulated Artemia (ZM ${ }^{\circledR}$ Prime) was also administered with a disposable Pasteur pipette at a mean concentration of 750,000 Artemia per liter of system water. Fish were fed in excess in all feeding events, i.e. were fed enough to feed continuously for 5 minutes with food still present in the water after this period of time.

\section{Fish measurements}

Individual fish counting was done at $3 \mathrm{dpf}, 30 \mathrm{dpf}, 60 \mathrm{dpf}$ and

Table 1: Feeding protocol for each feeding regimen and feed group after $6 \mathrm{dpf}$ (i.e. first day of exogenous feeding).

\begin{tabular}{|c|c|c|c|c|}
\hline & dpf & Skretting ${ }^{\circledR}$ Gemma Micro & Zeigler $^{\circledR}$ Larval AP100 & $\mathbf{Z} \mathbf{M}^{\circledR}$ \\
\hline \multirow{3}{*}{ Dry only } & 6-11 & $4 \times 75$ & $4 \mathrm{x}<100$ & $4 \times 00$ \\
\hline & $12-30$ & $4 \times 150$ & $4 \times 100-150$ & $4 \times 100$ \\
\hline & $31-60$ & $4 \times 150$ & $4 \times 150-250$ & $4 \times 200$ \\
\hline \multirow{4}{*}{ Dry + live } & $6-8$ & $4 \times 75$ & $4 \mathrm{x}<100$ & $4 \times 00$ \\
\hline & $9-11$ & $\begin{array}{l}2 \times 75 \\
2 \times 6 \text { drops of artemia }\end{array}$ & $\begin{array}{l}2 \mathrm{x}<100 \\
2 \mathrm{x} 6 \text { drops of artemia }\end{array}$ & $\begin{array}{l}2 \times 00 \\
2 \times 6 \text { drops of artemia }\end{array}$ \\
\hline & $12-30$ & $\begin{array}{l}2 \times 150 \\
2 \times 2 \mathrm{ml} \text { of artemia }\end{array}$ & $\begin{array}{l}2 \times 100-150 \\
2 \times 2 \mathrm{ml} \text { of artemia }\end{array}$ & $\begin{array}{l}2 \times 100 \\
2 \times 2 \mathrm{ml} \text { of artemia }\end{array}$ \\
\hline & $31-60$ & $\begin{array}{l}2 \times 150 \\
2 \times 5 \mathrm{ml} \text { of artemia }\end{array}$ & $\begin{array}{l}2 \times 150-250 \\
2 \times 5 \mathrm{ml} \text { of artemia }\end{array}$ & $\begin{array}{l}2 \times 200 \\
2 \times 5 \mathrm{ml} \text { of artemia }\end{array}$ \\
\hline \multirow{3}{*}{ Control } & $6-8$ & $4 \times 75$ & $4 \mathrm{x}<100$ & $4 \times 00$ \\
\hline & $9-11$ & $\begin{array}{l}2 \times 75 \\
2 \times 6 \text { drops of artemia }\end{array}$ & $\begin{array}{l}2 \mathrm{x}<100 \\
2 \mathrm{x} 6 \text { drops of artemia }\end{array}$ & $\begin{array}{l}2 \times 00 \\
2 \times 6 \text { drops of artemia }\end{array}$ \\
\hline & $31-60$ & $4 \times 5 \mathrm{ml}$ of artemia & $4 \times 5 \mathrm{ml}$ of artemia & $4 \times 5 \mathrm{ml}$ of artemia \\
\hline
\end{tabular}


$90 \mathrm{dpf}$. The 3-dpf counting was done under a light stereoscope whereas subsequent counts were done using a fish net.

Fish length was measured at $45 \mathrm{dpf}, 60 \mathrm{dpf}, 75 \mathrm{dpf}$ and 90 dpf. Each tank was photographed three times with a SONY® SteadyShot (DSC-W510) and the best photo was used to measure the fork length (from snout to tail split) in ten randomly chosen individuals (except in tanks with a density lower than 10 individuals, in which case all available individuals were measured) using Image J software (v. 1.45s, Wayne Rasband National Institutes of Health, USA).

\section{Density control}

Embryos were divided in 50 individuals per dish and at $6 \mathrm{dpf}$ vested to $3.5 \mathrm{~L}$ tanks (density of $\sim 14 \mathrm{fish} / \mathrm{L}$ ). At $30 \mathrm{dpf}$ all fish were counted and tanks holding more than 25 individuals were divided in two tanks for a maximum density of $\sim 7$ fish/L in order to decrease the influence of density in the overall results.

\section{Accessing reproductive parameters}

Starting at $75 \mathrm{dpf}$ and once every other week until $180 \mathrm{dpf}$ or until six successful matings (i.e. egg laying), all groups were set up to cross, one male and one female per breeding tank. In the next morning, eggs were collected and counted at $0 \mathrm{dpf}$ to determine the number of live fertilized eggs and at $3 \mathrm{dpf}$ to determine embryo survival.

\section{Statistical analysis}

All data was analyzed with the software IBM SPSS Statistics (v. 21, SPSS Inc., Chicago, IL). After assumption verification, t-student and one-way ANOVA test were used. Effect dimension was evaluated by $\eta 2 p$ and when significant differences were observed, they were analyzed with the Tukey's HSD test in order to perceive which groups differ. Significant statistical effects were considered for results with $\mathrm{p}$-value $<0.05$.

\section{Results}

Results were initially treated individually for the three fish groups (wild-type, mutant and transgenic). No differences were detected showing that fish strains do not significantly influence results (data not shown). Thus, all results for these fish groups were pooled and subsequently treated as a single group.

\section{Survival rates}

As depicted in Figure 1a, at 30dpf in the dry only diet group, significant differences were observed between tanks fed with Gemma Micro and ZM $(F(2,15)=4,615 ; p=0,027 ; \eta 2 p=0,381$; $\pi=0,689$ ) with Gemma Micro performing best (41\% survival) and ZM performing worst (3\% survival). All feed brands in the control group showed significant differences when compared amongst each other $(F(2,15)=30,78 ; p<0,001 ; \eta 2 p=0,78 ; \pi=1)$ revealing the influence of each dry feed as first feed, again with Gemma Micro showing the best survival (71\%) and Larval AP100 performing worst (25\%). Although ZM is not suitable alone to sustain growth, it does well as first feed (53\% survival). In the tanks fed with a combination of dry+live food, no significant differences were observed $(F(2,15)=0,101 ; p=0,905 ; \eta 2 p=0,013$; $\pi=0,063$ ) and the survival rates were generally increased except for Gemma Micro that showed a more sustained behavior between feeding regimens.

Similar to $30 \mathrm{dpf}$, at $90 \mathrm{dpf}$ (Figure 1b) the differences in survival rates between Gemma Micro and ZM in the dry only diet group $(F(2,15)=10,021 ; p=0,002 ; \eta 2 p=0,572 ; \pi=0,959)$ and between all of the feeds in the control group $(F(2,15)=35,09$; $\mathrm{p}<0,001 ; \eta 2 \mathrm{p}=0,751 ; \pi=1$ ) were sustained. In tanks fed with a combination of dry live feed, no significant differences were observed in the $90 \mathrm{dpf}$ survival rates $(\mathrm{F}(2,15)=0,115 ; \mathrm{p}=0,892$; $\eta 2 p=0,015 ; \pi=0,065$ )

Survival rates: dry only - Gemma Micro 41\%, Larval AP100 19\%, ZM 3\%; dry live - Gemma Micro 63\%, Larval AP100 61\%, ZM 58\%; control - Gemma Micro 71\%, Larval AP100 25\%, ZM $53 \%$.

\section{Growth}

At 45dpf (Figure 2a), in the dry only feeding group, Gemma Micro fed fish showed a significantly faster growth as compared with the other two brands $(F(2,100)=60,119$; $\mathrm{p}<0,001 ; \eta 2 \mathrm{p}=0,546 ; \pi=1$ ). Complemented with artemia (dry live group),Larval AP100 showed a slight, though significant, slower growth $(F(2,177)=4,931 ; p=0,008 ; \eta 2 p=0,053 ; \pi=0,803)$ than the other two which behaved similarly. In the control group there were significant differences between Gemma Microand Larval
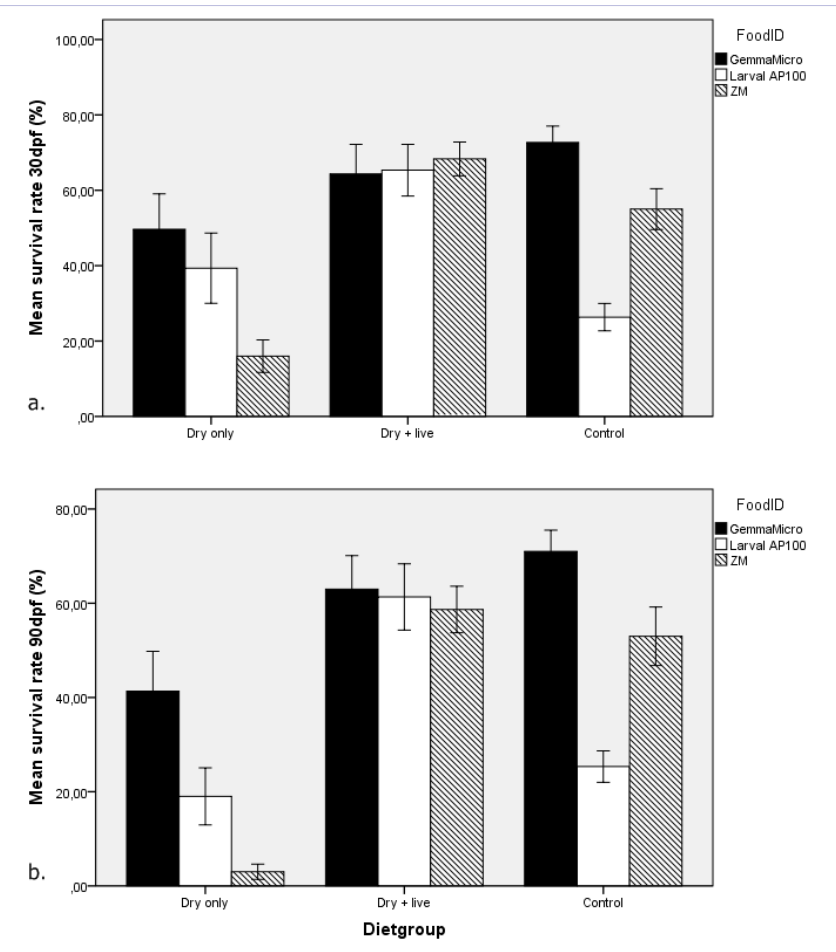

Figure 1: Fish survival rates: a) 30 days post-fertilization (dpf); b) 90 days post-fertilization. Error bars: \pm standard error of the mean. 

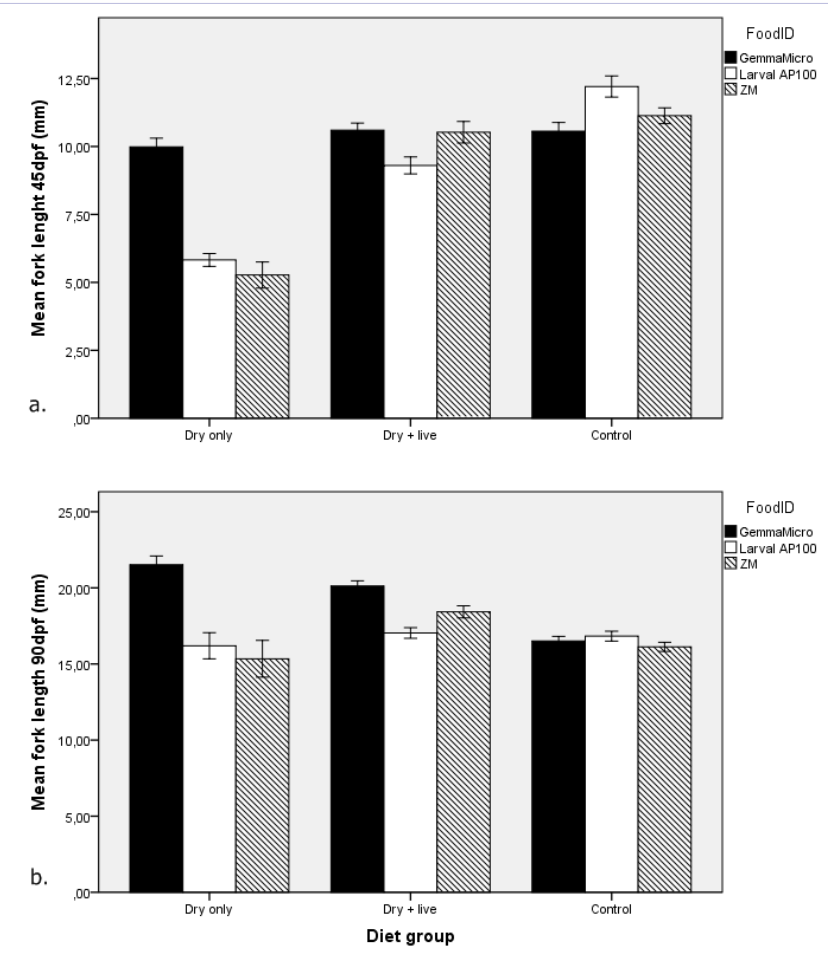

Figure 2: Fish growth assessment by mean fork length (mm): a) 45 days post-fertilization; b) 90 days post-fertilization. Error bars: \pm standard error of the mean.

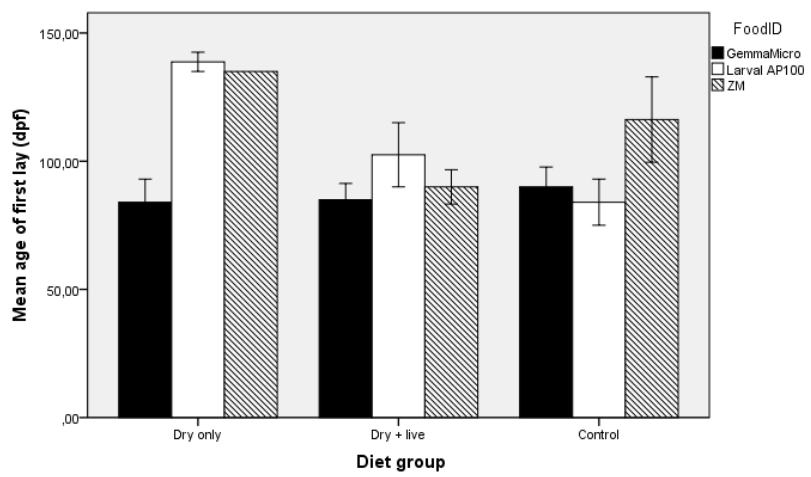

Figure 3: Mean fish age (day's post-fertilization) of first egg lay. Error bars: \pm standard error of the mean.

AP100 $(F(2,166)=6,042 ; p=0,003 ; \eta 2 p=0,068 ; \pi=0,879)$ groups although Larval AP100 showed a slight faster growth.

At 90 dpf (Figure 2b), in the dry only group, growth results were similar to $45 \mathrm{dpf}$, with Gemma Micro-fed fish continuing to grow faster than the ones fed with the other two brands $(F(2,85)=18,301 ; p<0,001 ; \eta 2 p=0,301 ; \pi=1)$. Growth rates were kept fairly constant for all feeds (compare lengths in Fig.2a and b). In the dry live group the overall tendency was sustained with Larval AP100-fed fish being slightly slower than the other two brands $(F(2,163)=19,472 ; p<0,001 ; \eta 2 p=0,193 ; \pi=1)$. The control group showed no differences between all feed brands in growth at $90 \mathrm{dpf}(\mathrm{F}(2,156)=5,030 ; \mathrm{p}=0,294 ; \eta 2 \mathrm{p}=0,016 ; \pi=0,266)$.
Average length: Dry only - Gemma Micro $=21,52 \mathrm{~mm}$, Larval AP100 =16,2mm, ZM =15,34mm; dry+live - Gemma Micro $=20,12 \mathrm{~mm}$, Larval AP100 $=17,03 \mathrm{~mm}, \mathrm{ZM}=18,42 \mathrm{~mm}$; control - Gemma Micro $=16,51 \mathrm{~mm}$, Larval AP100 $=16,83 \mathrm{~mm}$, ZM $=16,12 \mathrm{~mm}$.

\section{Breeding performance and Sexual maturity}

In Figure 3 we show the age at which fish laid their first eggs upon being set in pair crosses after sexual dimorphism has been visually observed, which age reflects sexual maturity. In the dry only feeding group, Gemma Micro produced significantly different results $(F(2,7)=17,703 ; p=0,002 ; \eta 2 p=0,835 ; \pi=0,99)$, with the age of fish sexual maturity being greatly reduced (less than $90 \mathrm{dpf}$ ) as compared to fish fed with the other two brands (more than 125 days). In the tanks fed with dry live, there were no significant differences betweenall feed brands $(F(2,15)=0,599$; $p=0,562 ; \eta 2 p=0,074 ; \pi=0,131$ ). This reveals that both Larval AP100 and ZM benefit from the complementation with artemia. In the control group differences between Larval AP100 and $\mathrm{ZM}$-fed fish $(\mathrm{F}(2,12)=4,14 ; \mathrm{p}=0,043 ; \eta 2 \mathrm{p}=0,408 ; \pi=0,615)$ were observed, again reflecting a negative impact of ZM as a first feed (as shown also for the survival) on the sexual maturity.

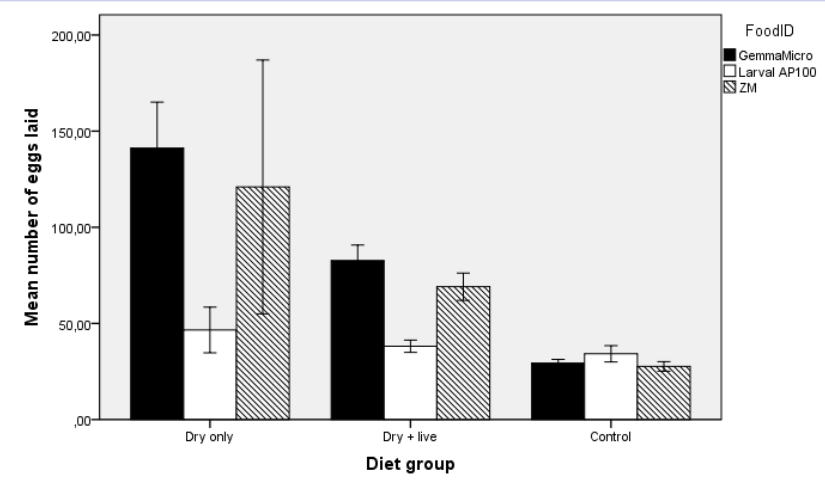

Figure 4: Mean number of fish eggs laid per breeding event. Error bars: \pm standard error of the mean.

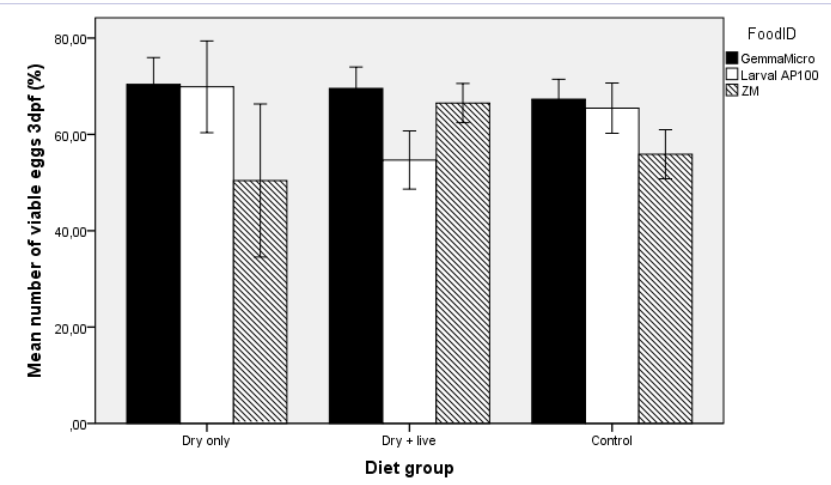

Figure 5: Embryo viability as the percentage of live embryos at 3 days post-fertilization compared to the number of eggs laid. Error bars: \pm standard error of the mean. 


\section{Fecundity}

As shown in Figure $4 \mathrm{a}$, the number of eggs laid were similar between the dry only group and the dry+live group. In the latter tanks fed with Larval AP100There were significant differences against the other two brands $(\mathrm{F}(2,104)=11,321$; $p<0,001 ; \eta 2 p=0,179 ; \pi=0,992)$. In the dry only group, there was no significant difference on the number of eggs laid, when the 3 groups were compared $(F(2,36)=2,78 ; p=0,075 ; \eta 2 p=0,134$; $\pi=0,513)$, most likely due to the very low number of embryos produced (and thus high variance) of the ZM assay. However, when comparing only Gemma Micro with Larval AP100, Gemma Micro-fed fish showed a significant higher fecundity (t (177) $=4,316 ; p<0,001)$. No significant differences were observed in the control group $(F(2,103)=1,228 ; p=0,297 ; \eta 2 p=0,023 ; \pi=0,262)$.

\section{Embryo viability}

As shown in Figure 4b, no significant differences were observed for all three diets regarding the percentage of viable embryos at $3 \mathrm{dpf}$ Figure 5 as compared with the amount of embryos with 1 $\operatorname{dpf}\left(\right.$ dry only $\left(\mathrm{F}(2,36)=0,446 ; \mathrm{p}=0,643 ; \eta_{\mathrm{p}}^{2}=0,024 ; \pi=0,117\right)$; dry + live $(F(2,103)=2,522 ; p=0,085 ; \eta 2 p=0,047 ; \pi=0,495)$; control $\left.\left(\mathrm{F}(2,103)=1,580 ; \mathrm{p}=0,211 ; \eta_{\mathrm{p}}^{2}=0,030 ; \pi=0,328\right)\right)$;

Average embryo viability: dry only - Gemma Micro 73\%, Larval AP100 74\%, ZM 77\%; dry+live - Gemma Micro 77\%, Larval AP100 58\%, ZM 74\%; control - Gemma Micro 71\%, Larval AP100 69\%, ZM 59\%.

\section{Discussion}

A previous study had already shown feeding trials presenting a better performance of Gemma Micro against another commercial dry feed in another fish species [48]. Our results have now shown the same better performance in zebrafish. On a feeding frequency study, this same diet had been already used in zebrafish with good results in adults without however being neither compared with any other feed nor tested in larval rearing [49]. In our facility conditions, Gemma Micro stood out as best performer in almost all assessed parameters, with fish presenting higher survival rates, shorter sexual maturity age and highest fecundity.

When using only live feed after $9 \mathrm{dpf}$ (control group), Larval AP100-fed fish showed a higher mortality at $30 \mathrm{dpf}(74 \%)$ as compared to the other diets. This probably suggests that the type of food intake from 5 to $9 \mathrm{dpfis}$ crucial to provide adequate nutrients for the larvae to grow and attain the necessary size to be able to eat Artemia. Larval AP100-fed fish showed also the lowest growth, oldest sexual maturity (102, 5 days) and lowest number of eggs laid per breeding pair event within the dry live group which further supports the idea that this feed does not offer adequate nutrients (as compared with the other two) to support zebrafish development even when complemented with Artemia.

Tanks fed under the dry only regimen with ZM showed the worst survival rates $(16 \%)$ and growth, which makes this feed a poor replacement for live feeds. In the control group it showed a high mortality rate in the survival assessment at $30 \mathrm{dpf}(45 \%)$ probably due to the same reason as Larval AP100: it did not provide enough nutrients for the larvae to growth and start eating Artemia. Regarding the breeding parameters in the control group, although ZM-fed fish had a slight poorer performance as compared with the other two brands, all feeds showed a very poor breeding performance, revealing that Artemia by itself is a poor diet for optimal breeding success regardless of the first feed. However, when dry and live feeds are combined, ZM showed results as good as Gemma Micro, suggesting that this feed may be suitable to complement, but not to replace, live feeding practices at later stages.

It should be noted that in this study no live feed was used from 5 to $9 \mathrm{dpf}$ in order to assess the impact of the dry feeds as first exogenous feed. However, if a live feed such as Paramecium or rotifers would be used as first feed, we believe that survival rates would be improved for all feeds, possibly allowing for a dry only feeding regimen after day 9 if using Gemma Micro in view of the results presented here.

Importantly, Larval AP100 and ZM-fed fish had both higher mortality rates than Gemma Micro, which resulted in a lower density, even with the density control measures taken. Density is known to affect reproductive performance [50] and lower densities can also make individuals grow faster than in higher densities which could account for the difference attenuation in both growth and reproductive performance within the control group. Interestingly, even with higher mortality rates in the dry only regimen, and consequently lower densities, fish fed with Larval AP100 and ZM did not surpass the ones fed with Gemma Micro.

Several studies in zebrafish fed with different commercial/ non commercial feeds had shown effects of different feeds and feeding regimens in reproductive performance [41, 49, 51, 52]. However, fish were fed with test feeds only after young juvenile or adult stages, thus not revealing the impact of feeding during development nor assessing the quality of dry feeds during larval rearing. Our study has shown that fish may attain sexual maturity as soon as $75 \mathrm{dpf}$ depending on the feed and feeding regimen. As expected, our results reflect the effect of the different feeding protocols in the reproductive performance through the number of eggs laid per breeding event, where fish fed with Gemma Micro produced the best results. However, no impact of the tested feeds was seen in the embryo viability, with $30-40 \%$ embryo mortality being observed for all feed types in all feeding regimens. Similar to what was discussed above for the survival, we believe that if a live feed would be used as first feed, this embryo mortality may be greatly reduced due to the impact on embryo quality [46]. We also analyzed the female/male ratio per tank but it resulted in no significant differences (data not shown).

We believe that the difference in composition of the 4 types of feed tested ( 3 dry feeds + Artemia, Table 2 ) is not likely to support 
Table 2: Nutritional composition for each tested feed. GemmaMicro and Larval AP100nutritionalcontent was the same for all granule sizes. "-" means no information available. Information gathered from products' labels.

\begin{tabular}{|c|c|c|c|c|c|}
\hline & \multirow{2}{*}{ Skretting ${ }^{\circledR}$ Gemma Micro } & \multirow{2}{*}{ Zeigler ${ }^{\circledR}$ Larval AP100 } & \multicolumn{3}{|l|}{$\mathbf{Z M}^{\circledR}$} \\
\hline & & & $\mathbf{0 0}$ & 100 & 200 \\
\hline Protein & $59 \%$ & $>55 \%$ & $52 \%$ & $55 \%$ & $60 \%$ \\
\hline FatandOils & $14 \%$ & $>15 \%$ & $12 \%$ & $13 \%$ & $14.5 \%$ \\
\hline Fiber & $0.2 \%$ & $<1.5 \%$ & $3 \%$ & $1 \%$ & - \\
\hline Ash & $13 \%$ & $<8 \%$ & $8 \%$ & $8 \%$ & $11.5 \%$ \\
\hline Calcium & $1.5 \%$ & - & - & - & - \\
\hline Sodium & $0.7 \%$ & - & - & - & - \\
\hline Moisture & - & $<12 \%$ & $7 \%$ & $7 \%$ & $7 \%$ \\
\hline
\end{tabular}

the significantly different data obtained in this study. Although we did not have access to the detailed composition of each feed, in particular the fatty acid n-3/n-6 ratio, the general content in protein and fatty acids is not dramatically different. However, Gemma Micro is produced by a new process substantially different from the other two dry feeds (and to the best of our knowledge all other dry feeds currently used in zebrafish) - the method called cold extrusion spherizer agglomeration production, a process of cold extrusion of raw materials. As stated by Skretting and others $[53,54]$, with this method the protein, carbohydrates, essential oils and vitamins save their own specific values without any loss, the nutrients being more digestible and stable in water. As a result, they either prevent water pollution in the tanks or the fish consume it with more desire and digest very easily, which may explain our results. Accordingly, and as a qualitative observation, tanks fed with Gemma Micro were recorded had having cleaner environments when compared with the other two brands.

Zebrafish had been previously reared successfully without any live feeds [41], however the diet was custom-formulated which imposes a difficulty to use it in others facilities. Our data have now shown relatively good results with an available commercial feed. Gemma Micro's performance when used alone shows moderate results during development (especially in survival rates), which in a large scale practice may have a negative impact suggesting that it may not be suitable to fully replace live feeds, at least during developmental stages. However, when compared with the other tested feeds, it appears as the most suitable feed to complement live feed protocols. Notwithstanding, Artemia did have a negative effect on Gemma Micro breeding performance Figure 4, which altogether points to the idea that Gemma Micro may be suitable to completely replace Artemia in adult zebrafish.

\section{Acknowledgments}

This work was supported by the Champalimaud Foundation. The authors wish to thank Joana Robalo for selecting M.F. to perform this study and also Sandra Martins and Joana Monteiro for critical reading of the manuscript.

\section{Conflict of Interest Statement}

The authors declare that the research was conducted in the absence of any commercial or financial relationships that could be construed as a potential conflict of interest.

\section{References}

1. Nüsslein-Volhard C. The zebrafish issue of Development. Development. 2012;139(22):4099-103. doi: 10.1242/dev.085217.

2. Müller B, Grossniklaus U. Model organisms - A historical perspective. J Proteomics. 2010;73(11):2054-63. doi: 10.1016/j.jprot.2010.08.002.

3. Grunwald DJ, Eisen JS. Headwaters of the zebrafish - emergence of a new model vertebrate. Nat Rev Genet. 2002;3(9):717-24.

4. Richard JKN, Müller P, Klein R. Life Cycle Stages and Length of Zebrafish (Danio rerio) Exposed to DDT. Journal of Health Science. 2004;50(3):220-225.

5. Boyle D, Amlund H, Lundebye AK, Hogstrand C, Bury NR. Bioavailability of a natural lead-contaminated invertebrate diet to zebrafish. Environ Toxicol Chem. 2010;29(3):708-14. doi: 10.1002/etc.61.

6. Leung LC, Wang GX, Mourrain P. Imaging zebrafish neural circuitry from whole brain to synapse. Front Neural Circuits. 2013;7:76. doi: 10.3389/fncir.2013.00076.

7. Arrenberg $\mathrm{AB}$, Driever W. Integrating anatomy and function for zebrafish circuit analysis. Front Neural Circuits. 2013;7:74. doi: 10.3389/fncir.2013.00074.

8. Portugues R, Feierstein CE, Engert F, Orger MB. Whole-Brain Activity Maps Reveal Stereotyped, Distributed Networks for Visuomotor Behavior. Neuron. 2014;81(6):1328-43. doi: 10.1016/j. neuron.2014.01.019.

9. Kermen F, Franco LM, Wyatt C, Yaksi E. Neural circuits mediating olfactory-driven behavior in fish. Front Neural Circuits. 2013;7:62. doi: 10.3389/fncir.2013.00062.

10. Morin C, de Souza Silva MA, Müller CP, Hardigan P, Spieler RE. Active avoidance learning in zebrafish (Danio rerio) - the role of sensory modality and inter-stimulus interval. Behav Brain Res. 2013;248:1413. doi: 10.1016/j.bbr.2013.04.009.

11. Maaswinkel H, Le X, He L, Zhu L, Weng W. Dissociating the effects of habituation, black walls, buspirone and ethanol on anxiety-like behavioral responses in shoaling zebrafish. A 3D approach to social behavior. Pharmacol Biochem Behav. 2013;108:16-27. doi: 10.1016/j. pbb.2013.04.009.

12. Ma ACH, Guo Y, He ABL, Leung AYH. Modeling Tumor Angiogenesis with Zebrafish. In: Vasculogenesis and Angiogenesis - from Embryonic Development to Regenerative Medicine. Chapter 7. Dan T. Simionescu and Agneta Simionescu Eds. 2011;133-144. 
13. Goessling W, North TE, Zon LI. New waves of discovery: modeling cancer in zebrafish. J Clin Oncol. 2007;25(17):2473-9.

14. Moshal KS, Ferri-Lagneau KF, Leung T. Zebrafish model: worth considering in defining tumor angiogenesis. Trends Cardiovasc Med. 2010;20(4):114-9. doi: 10.1016/j.tcm.2010.10.001.

15. Howe K, Clark MD, Torroja CF, Torrance J, Berthelot C, Muffato M, et al. The zebrafish reference genome sequence and its relationship to the human genome. Nature. 2013;496(7446):498-503. doi: 10.1038/ nature12111.

16. Yokobori E, Azuma M, Nishiguchi R, Kang KS, Kamijo M, Uchiyama M, et al. Neuropeptide Y stimulates food intake in the Zebrafish, Danio rerio. J Neuroendocrinol. 2012;24(5):766-73. doi: 10.1111/j.13652826.2012.02281.x.

17. Maddison LA, Chen W. Nutrient excess stimulates $\beta$-cell neogenesis in zebrafish. Diabetes. 2012;61(10):2517-24

18. Flynn EJ, Trent CM, Rawls JF. Ontogeny and nutritional control of adipogenesis in zebrafish (Danio rerio). J Lipid Res. 2009;50(8):164152. doi: 10.1194/jlr.M800590-JLR200.

19. Ulloa PE, Iturra P, Neira R, Araneda C. Zebrafish as a model organism for nutrition and growth: towards comparative studies of nutritional genomics applied to aquacultured fishes. Rev Fish Biol Fisheries. 2011;21:649-666.

20. Ulloa PE, Medrano JF, Feijoo CJ. Zebrafish as animal model for aquaculture nutrition research. Front Genet. 2014;5:313. doi: 10.3389/fgene.2014.00313.

21. Dahm R, Geisler R. Learning from small fry: the zebrafish as a genetic model organism for aquaculture fish species. Mar Biotechnol (NY). 2006;8(4):329-45.

22. Lieschke GJ, Currie PD. Animal models of human disease: zebrafish swim into view. Nat Rev Genet. 2007;8(5):353-67.

23. Willemsen R, Hasselaar W, Linde H, Bonifati V. Zebrafish as a new model organism for Parkinson's disease. In Proceedings of Measuring Behavior. Spink AJ, Ballintijn MR, Bogers ND, et al. Eds. 2008;50-51.

24. Meeker ND, Trede NS. Immunology and zebrafish: spawning new models of human disease. Dev Comp Immunol. 2008;32(7):745-57. doi: 10.1016/j.dci.2007.11.011.

25. You M-S, Jiang Y-J. Drug Discovery Using Zebrafish. Drug Discov. $2007 ; 1-2$.

26. Goldsmith JR, Jobin C. Think small: zebrafish as a model system of human pathology. J Biomed Biotechnol. 2012;2012:817341. doi: $10.1155 / 2012 / 817341$.

27. Gerhard GS. Comparative aspects of zebrafish (Danio rerio) as a model for aging research. Exp Gerontol. 2003;38(11-12):1333-41.

28. Raya A, Consiglio A, Kawakami Y, Rodriguez-Esteban C, IzpisúaBelmonte JC. The zebrafish as a model of heart regeneration. Cloning and Stem Cells. 2004;6:345-51.

29. Lawrence C. The husbandry of zebrafish (Danio rerio): A review. Aquaculture 2007; 269: 1-20.

30. Kaushik S, Georga I, Koumoundouros G. Growth and body composition of zebrafish (Danio rerio) larvae fed a compound feed from first feeding onward: toward implications on nutrient requirements. Zebrafish. 2011; 8(2):87-95. doi: 10.1089/zeb.2011.0696.

31. Westerfield M. The Zebrafish Book: A Guide for the Laboratory Use of Zebrafish (Danio rerio). Eugene: University of Oregon Press. 2007; 3.1-3.2.
32. Wilson C. Aspects of larval rearing. ILAR J. 2012; 53(2):169-78. doi: 10.1093/ilar.53.2.169.

33. Watts SA, Powell M, D'Abramo LR. Fundamental approaches to the study of zebrafish nutrition. ILAR J. 2012; 53(2):144-60. doi: 10.1093/ ilar.53.2.144.

34. Brendan C, Delbos MS. Role of Live Feeds in Zebrafish Culture. 1-5.

35. Markovich ML, Rizzuto NV, Brown PB. Diet affects spawning in zebrafish. Zebrafish. 2007 spring; 4(1):69-74.

36. Lawrence FC, Westerfield M, Zon LI. Infrastructure. 2011;104: 430452.

37. Reed B, Jennings M. Guidance on the housing and care of Zebrafish Danio rerio. 2011; Available at http://www.scilifelab.se/wp-content/ uploads/2013/10/Guidance-zebrafish.pdf

38. Harper C, Lawrence C. The laboratory zebrafish. CRC Press. Mark A. Suckow Ed. 2011. 274.

39. Mehrad B, Jafaryan H, Taati MM. Assessment the effects of dietary vitamin $\mathrm{E}$ on growth performance and reproduction of zebrafish, Danio rerio (Pisces, Cyprinidae). J. Oceanogr. Mar. Sci. 2012; 3: 1-7.

40.Craig MP, Desai MB, Olukalns KE, Afton SE, Caruso JA, Hove JR. Unsupplemented Artemia Diet Results in Reduced Growth and Jaw Dysmorphogenesis in Zebrafish. 2006; Available at http://cdn. intechopen.com/pdfs-wm/27102.pdf.

41. Siccardi AJ, Garris HW, Jones WT, Moseley DB, D’Abramo LR, Watts SA. Growth and survival of zebrafish (Danio rerio) fed different commercial and laboratory diets. Zebrafish. 2009; 6(3):275-80. doi: 10.1089/zeb.2008.0553.

42.Gonzales JM. Preliminary evaluation on the effects of feeds on the growth and early reproductive performance of zebrafish (Danio rerio). J Am Assoc Lab Anim Sci. 2012; 51(4):412-7.

43. Ulloa PE, Peña AA, Lizama CD, et al. Growth Response and Expression of Muscle Growth-Related Candidate Genes in Adult Zebrafish Fed Plant and Fishmeal Protein-Based Diets. Zebrafish. 2013;10(1):99109. doi: 10.1089 /zeb.2012.0823.

44. Yossa R, Sarker PK, Karanth S, Ekker M, Vandenberg GW. Effects of dietary biotin and avidin on growth, survival, feed conversion, biotin status and gene expression of zebrafish Danio rerio. Comp Biochem Physiol B Biochem Mol Biol. 2011;160(4):150-8. doi: 10.1016/j. cbpb.2011.07.005.

45. Siccardi AJ, Padgett-Vasquez S, Garris HW, Nagy TR, D’Abramo LR, Watts SA. Dietary strontium increases bone mineral density in intact zebrafish (Danio rerio): a potential model system for bone research. Zebrafish. 2010; 7(3):267-73. doi: 10.1089/zeb.2010.0654.

46. Best J, Adatto I, Cockington J, James A, Lawrence C. A novel method for rearing first-feeding larval zebrafish: polyculture with Type L saltwater rotifers (Brachionus plicatilis). Zebrafish. 2010; 7(3):28995. doi: 10.1089/zeb.2010.0667.

47. Akerboom J, Chen TW, Wardill TJ, Tian L, Marvin JS, Mutlu S, et al. Optimization of a GCaMP Calcium Indicator for Neural Activity Imaging. J Neurosci. 2012; 32(40):13819-40. doi: 10.1523/ JNEUROSCI.2601-12.2012.

48. Curnow J, King J, Partridge G, Kolkovski S. Effects of two commercial microdiets on growth and survival of barramundi (Lates calcarifer Bloch) larvae within various early weaning protocols. Aquac. Nutr. 2006; 12: 247-255. 
49. Lawrence C, Best J, James A, Maloney K. The effects of feeding frequency on growth and reproduction in zebrafish (Danio rerio). Aquaculture.2012; 368-369, 103-108.

50. Castranova D. Lawton A, Lawrence C, et al. The effect of stocking densities on reproductive performance in laboratory zebrafish (Danio rerio). Zebrafish. 2011;8(3):141-6. doi: 10.1089/zeb.2011.0688.

51. Gonzales JM, Law SH. Feed and Feeding Regime Affect Growth Rate and gonadosomatic Index of Adult Zebrafish (Danio rerio). Zebrafish. 2013; 10(4):532-40. doi: 10.1089/zeb.2013.0891.
52. Markovich ML, Rizzuto NV, Brown PB. Diet affects spawning in zebrafish. Zebrafish. 2007 spring; 4(1):69-74.

53. Colin Mair. New! Tech feature: cold extrusion. 2004. Available at: http://www.aquafeed.com/news/headline-news-article/601/NEWTECH-FEATURE-COLD-EXTRUSION/

54. Available at: http://www.gemmafish.com/Content/2/11/ABOUTGEMMA.aspx 